LA-UR-01-3505

Approved for public release; distribution is unlimited.

\section{Effects of in-plane strain on orbital ordering and magnetism in $\mathrm{LaMnO}_{3}$ thin film}

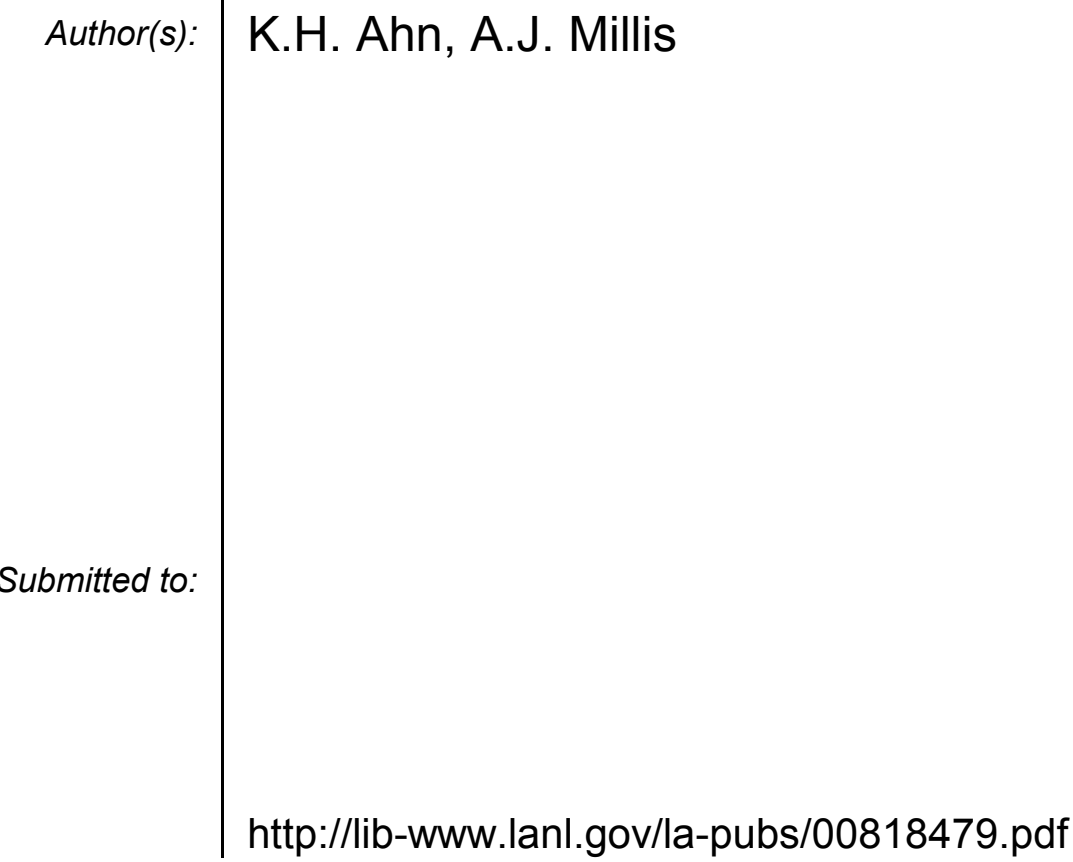




\title{
Effects of in-plane strain on orbital ordering and magnetism in $\mathrm{LaMnO}_{3}$ thin film
}

\author{
K. H. Ahn ${ }^{a, *}$ A. J. Millis ${ }^{b}$ \\ a Theoretical Division, T-11, Los Alamos National Laboratory, Los Alamos, New \\ Mexico, 87545 , USA \\ ${ }^{\mathrm{b}}$ Center for Materials Theory, Department of Physics and Astronomy, Rutgers \\ University, Piscataway, New Jersey, 08854, USA
}

\begin{abstract}
The effects of the in-plane strain on the orbital and magnetic properties of $\mathrm{LaMnO}_{3}$ thin films are calculated using an elastic energy expression and a tightbinding Hamiltonian with electron-lattice coupling. Tensile uniaxial strain of the order of $2 \%$, which is the order of magnitude of those induced in thin films by lattice mismatch with substrates, is found to change the orbital state and magnetic ground state significantly.
\end{abstract}

Key words:

orbital ordering, perovskite manganite, novel d-electron materials

Since most of the future technological applications of the 'colossal' magnetoresistive manganese perovskites would require thin films on substrates, it is important to understand the effects of strains induced by substrates. Because of the strong Jahn-Teller electron-lattice coupling, it is expected that even relatively small in-plane strains may result in observable effects on the electronic properties of these materials, which has been shown by recent experiments [1]. In this work, we study the effects of uniaxial strains in $\mathrm{LaMnO}_{3}$, which has a $(\pi, \pi, 0)$ type JT distortion (or orbital ordering) below $750 \mathrm{~K}$, and A-type (or $(0,0, \pi)$ type) antiferromagnetic (AF) spin ordering below $140 \mathrm{~K}$.

The total elastic energy per Mn ion we will consider is

$$
E_{\text {tot }}^{\text {elastic }} / N_{M n}=\left(E_{u}+E_{s}+E_{J T}+E_{a n h}\right) / N_{M n} .
$$

* Corresponding Author: Phone: (505) 667-8648, Fax: (505) 665-4063, Email: ahn@viking.lanl.gov

Preprint submitted to SCES'2001: Version $1 \quad 30$ August 2001 
$E_{u}$ and $E_{s}$ are the elastic energies due to the uniform strains and the $(\pi, \pi, 0)$ staggered strains, respectively: $E_{u} / N_{M n}=K_{B} Q_{1 u}^{2} / 2+K^{*}\left(Q_{2 u}^{2}+Q_{3 u}^{2}\right) / 2$, $E_{s} / N_{M n}=K_{2 s} Q_{2 s}^{2} / 2+K_{3 s} Q_{3 s}^{2} / 2$, where $Q_{1 u}=a_{0}\left(e_{x x}+e_{y y}+e_{z z}\right) / \sqrt{3}, Q_{2 u}=$ $a_{0}\left(e_{x x}-e_{y y}\right) / \sqrt{2}, Q_{3 u}=a_{0}\left(2 e_{z z}-e_{x x}-e_{y y}\right) / \sqrt{6}, Q_{2 s}=a_{0}\left(v_{s x}-v_{s y}\right) / \sqrt{2}$, and $Q_{3 s}=a_{0}\left(2 v_{s z}-v_{s x}-v_{s y}\right) / \sqrt{6}, v_{s a}$ is the $(\pi \pi 0)$ amplitude of $v_{\vec{i}}^{a}=u_{\vec{i}}^{a}-u_{\vec{i}-\hat{a}}^{a}$, and $u_{\vec{i}}^{a}$ is the displacement along the $a$ direction of an oxygen ion located between Mn ion at $\vec{i}$ and $\vec{i}+\hat{a}$. $E_{J T}$ represents the Jahn-Teller coupling between Mn $e_{g}$ orbital state and the lattice distortion: $E_{J T} / N_{M n}=-\sqrt{3 / 2} \lambda\left[\cos 2 \theta_{1}\left(Q_{3 u}+\right.\right.$ $\left.\left.Q_{3 s}\right)+\sin 2 \theta_{1}\left(Q_{2 u}+Q_{2 s}\right)+\cos 2 \theta_{2}\left(Q_{3 u}-Q_{3 s}\right)+\sin 2 \theta_{2}\left(Q_{2 u}-Q_{2 s}\right)\right] / 2$, where $\theta_{1,2}$ represents $\mathrm{Mn} e_{g}$ orbital state on the two sublattices, $\left|\theta_{\vec{i}}\right\rangle=\cos \theta_{\vec{i}} \mid 3 z^{2}-r^{2}>$ $+\sin \theta_{\vec{i}} \mid x^{2}-y^{2}>E_{a n h}$ is the anharmonic elastic energy between the nearest neighbor Mn and O ions: $E_{a n h}=4 A a_{0}^{3} / \sqrt{3} \sum_{i, a}\left(e_{a a} / 2+u_{i}^{a}-\delta_{i}^{a}\right)^{3}+\left(\delta_{i}^{a}-u_{i-a}^{a}-\right.$ $\left.e_{a a} / 2\right)^{3}$, where $\vec{\delta}_{\vec{i}}$ represents the Mn ion displacement vector at site $\vec{i}$.

We minimize $E_{u}+E_{s}+E_{J T}$, about the orbital state (This gives $\tan \left(2 \theta_{1,2}\right)=$ $\pm Q_{2 s} / Q_{3 u}$.) and lattice distortions and then treat $E_{a n h}$ as a perturbation. From this we obtain the total energy: $E_{\text {tot }} / N_{M n}=K_{B} Q_{1 u}^{2} / 2+K^{*} Q_{3 u}^{2} / 2+$ $K_{2 s} Q_{2 s}^{2} / 2-\sqrt{3 / 2} \lambda \sqrt{Q_{2 s}^{2}+Q_{3 u}^{2}}+A Q_{2 s}^{2}\left(Q_{1 u}-Q_{3 u} / \sqrt{2}\right)$. For thin films with in-plane strains, we calculate the lattice distortions in the following way: We assume that $(\pi \pi 0)$ distortion pattern is favored even in strained films, and the film is thin enough to make the epitaxy perfect. Therefore, $e_{x x}$ and $e_{y y}$ are determined by substrates, and other lattice distortions and orbital states, $\theta_{1,2}$, can be found by minimizing $E_{t o t}(\pi, \pi, 0)$. Parameters of the model are determined from experiments, as explained in Ref. [2].

$\theta_{1}$ versus in-plane strain, $e_{\|}$, (Here, we assume square lattice for substrate.) is shown in Fig. 1. The change in $\theta_{1}$ is about $\pm 5^{\circ}-15^{\circ}$. For tensile strains, orbital states approach towards $\left|x^{2}-y^{2}\right\rangle$. For compressive strains, towards $\mid 3 z^{2}-r^{2}>$. This can be understood from the fact that in $\theta_{1}, \theta_{2}=\left|x^{2}-y^{2}\right\rangle$ state, the x-y plane Mn-O-Mn distance tends to be farthest, and in $\theta_{1}, \theta_{2}$ $=\left|3 z^{2}-r^{2}\right\rangle$, shortest. Since the change of $\theta_{1}$ by $30^{\circ}$ would correspond to changing the orbital state from $\mid 3 x^{2}-r^{2}>$ to $\left|x^{2}-y^{2}\right\rangle$, the $15^{\circ}$ change predicted for $2 \%$ strain is a very large change of the orbital state, which is the result of the strong electron-lattice coupling combined with the small effective modulus $K^{*}-K_{2 s}[2]$.

Once the lattice distortions for thin films are determined from the elastic energy, then we use a tight-binding Hamiltonian with the Hartree-Fock approximation of the on-site Coulomb interaction to calculate total $e_{g}$ electronic energy for different magnetic orderings. The tight-binding Hamiltonian for Mn $e_{g}$ electron we use is explained in detail in Ref. [3]. The magnetic energy in $\mathrm{LaMnO}_{3}$ has two contributions: one from an isotropic $t_{2 g}$ derived corespin core-spin interaction, and one from the motion of the $e_{g}$ electrons [4]: $E_{\text {mag }}=J_{t 2 g} \sum_{\vec{i}, \hat{\delta}} \hat{S}_{c, \vec{i}} \cdot \hat{S}_{c, \vec{i}+\hat{\delta}}+E_{t b}\left[\left\{\hat{S}_{c}\right\}\right]$. Here $J_{t 2 g}$ is fixed by the observation 
that $\mathrm{CaMnO}_{3}$ ( the end-member with no $e_{g}$ electrons) is an antiferromagnet with $T_{N} \approx 120 \mathrm{~K} . E_{t b}$ is the energy of the $e_{g}$ electron calculated by the tight binding model, $<H_{\text {tot }}^{e g}>$.

For the lattice distortions in strained films obtained above, we calculate the $e_{g}$ electronic energies for the competing magnetic orderings. The results are shown in Fig. 2. It shows that as the tensile strain is applied, the purely AF ordering is favored over the A-AF, by about $50 \mathrm{meV}$, which will be increased further if we add $t_{2 g}$ exchange energy. This change is larger than the $T_{N} \sim$ $10 \mathrm{meV}$ for bulk $\mathrm{LaMnO}_{3}$. Therefore, the results suggest that the magnetic order can be changed to pure AF by $2 \%$ tensile strain. The main reason for this is the change of the hybridization energy, $-2 t^{2} / \Delta$, where $t$ is the hopping amplitude between filled and empty levels with energy difference $\Delta$. For the $3 x^{2}-r^{2} / 3 y^{2}-r^{2}$ orbital state in bulk $\mathrm{LaMnO}_{3}$, the hopping between the lower JT level $\left(3 x^{2}-r^{2}\right)$ at site $\vec{i}$ and the upper JT level $\left(z^{2}-x^{2}\right)$ at site $\vec{i}+\hat{x}$ is $t=\sqrt{3} t_{o} / 2$. This hybridization makes A-AF favored in bulk orbital ordering. As the orbital ordering changes towards $x^{2}-y^{2} / x^{2}-y^{2}$ by tensile strain, the hopping between lower $\left(x^{2}-y^{2}\right)$ and upper $\left(3 z^{2}-r^{2}\right)$ JT levels decreases $\left(t=\sqrt{3} t_{o} / 4\right)$. At this expense, the hopping between the two lower JT levels in different spin manifolds, which is possible for pure AF, increases. The change of the orbital state by $2 \%$ strain is large enough to induce a pure AF ground state. Since $t_{0} \sim 0.6 \mathrm{eV}, \Delta \sim 3-7 \mathrm{eV}$, the typical change of the kinetic energy is a fraction of $100 \mathrm{meV}$, which agrees with the calculation.

In summary, we found that $\pm 2 \%$ strain in $\mathrm{LaMnO}_{3}$ can induce significant changes in the $e_{g}$ orbital states and magnetic properties. This result shows that the strong coupling between electron and lattice is crucial to understand the effects of the substrate-induced strain in thin film perovskite manganites. This work is supported by NSF-DMR-9705182, the University of Maryland and Rutgers University MRSEC, and U.S. DOE. 


\section{References}

[1] J. O'Donnell et al., Phys. Rev. B 54, 6841 (1996); Y. Suzuki et al., Appl. Phys. Lett. 71, 140 (1997); B. S. Teo et al., J. Appl. Phys. 83, 7157 (1998); A. J. Millis et al., J. Appl. Phys. 83, 1588 (1998).

[2] K. H. Ahn and A. J. Millis, Phys. Rev. B (To be published).

[3] K. H. Ahn and A. J. Millis, Phys. Rev. B. 61, 13545 (2000).

[4] I. V. Solovyev et al., Phys. Rev. Lett. 76, 4825 (1996); O. N. Mryasov et al., Phys. Rev. B 56, 7255 (1997). 


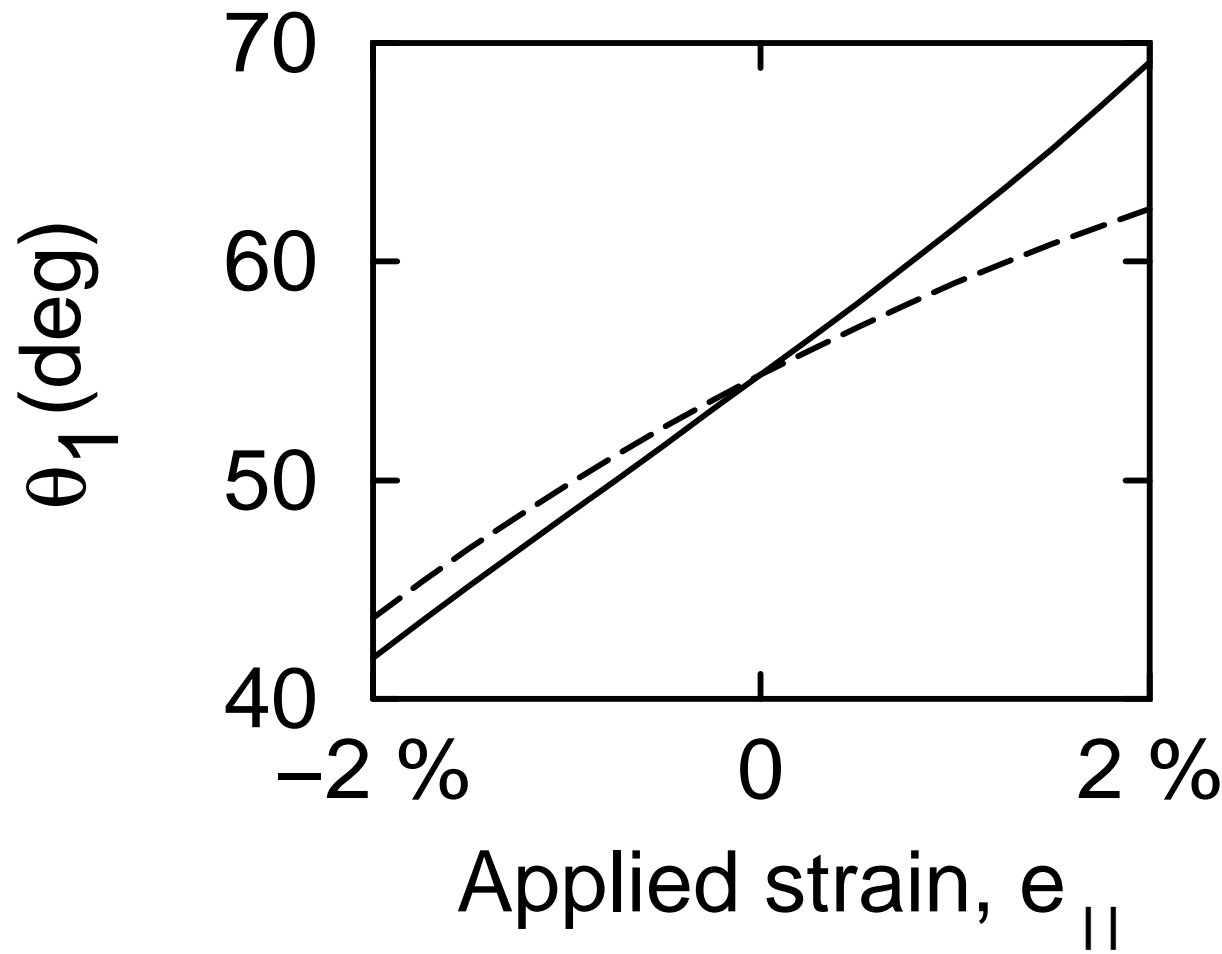

Fig. 1. $\theta_{1}$ versus $e_{\|}$: The solid and dashed lines are for the parameter sets determined from two experiments. See Ref. [2] for detail. 


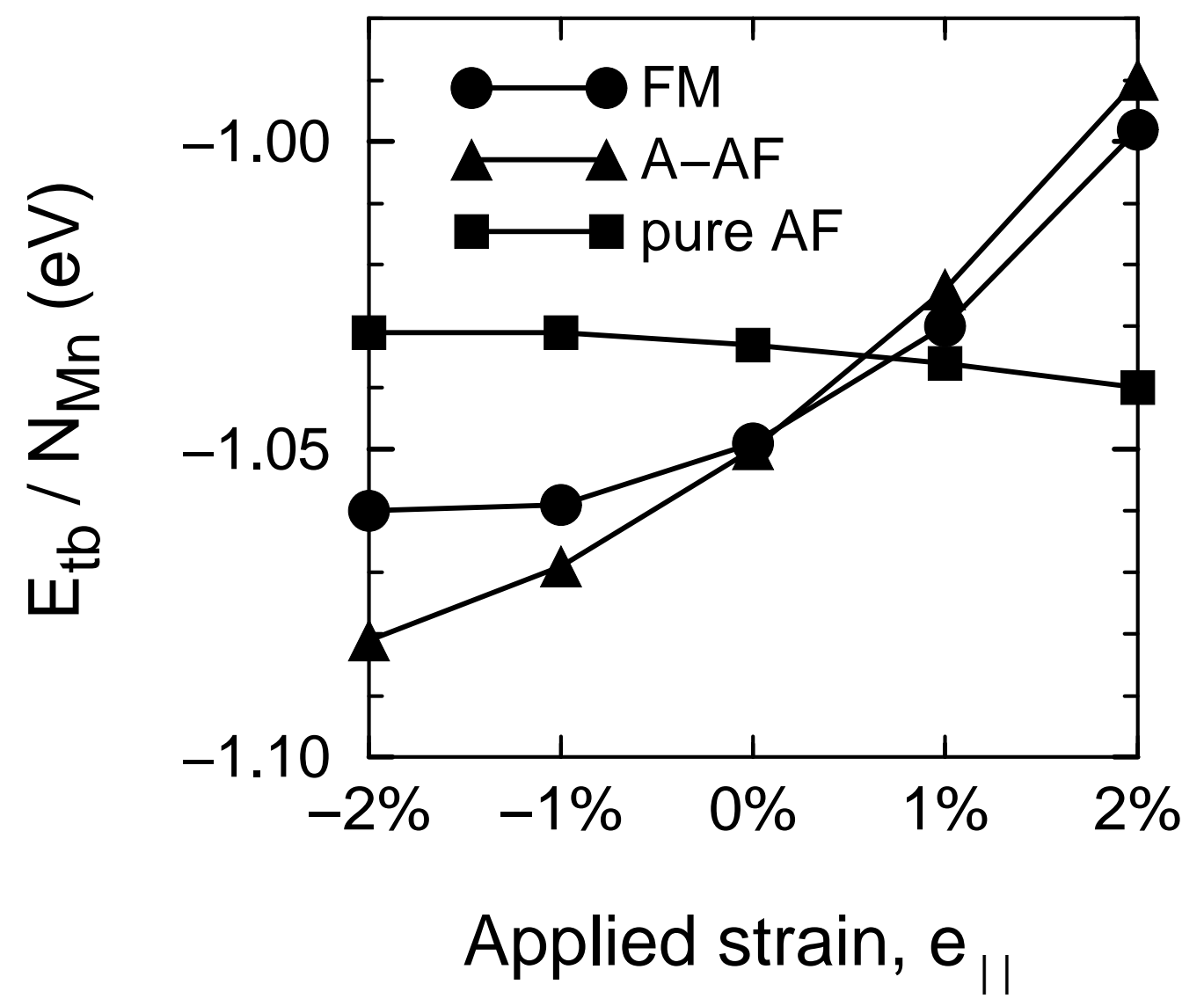

Fig. 2. Total $e_{g}$ electronic energy versus applied strain, for ferromagnetic, A-type antiferromagnetic, and purely antiferromagnetic core spin configurations. We use $t_{o}=0.622 \mathrm{eV}, 2 J_{H} S_{c}=3.7 \mathrm{eV}, \lambda=1.7 \mathrm{eV} / \AA, U=1.2 \mathrm{eV}$, and lattice distortions for the solid lines in Fig. 1. 